\title{
The Journal of Knee Surgery
}

\section{Barbed Sutures in TKA: Are they really useful? A Randomized Controlled Trial}

\begin{tabular}{|c|c|}
\hline Journal: & Journal of Knee Surgery \\
\hline Manuscript ID & JKS-17-Nov-0262-OA \\
\hline Manuscript Type: & Original Article \\
\hline Specialty Area: & Knee Arthroplasty, Barbed Suture, Controlled Randomized Trial \\
\hline Abstract: & $\begin{array}{l}\text { Purpose and Hypothesis } \\
\text { Bidirectional Barbed sutures (BBS) have recently been investigated in TKA } \\
\text { The contrasting results from the scarce literature suggest that BBS are } \\
\text { safe, save time and money and give results comparable to traditional } \\
\text { sutures. The purpose of the study is to test the real effect of BBS on } \\
\text { closure time in TKA and assess the functional results as well as the } \\
\text { complications related to them. } \\
\text { Methods } \\
\text { It was a randomized controlled trial (RCT). Eighty-five patients undergoing } \\
\text { primary TKA were assigned to receive traditional closure with Vycril (V- } \\
\text { Group) or with BBS (Q-group). The exclusion criteria were significant } \\
\text { coronal deformity, flexion contracture or the need for stem and/or } \\
\text { augmentation. The closure time for the capsule and subcutaneous layer } \\
\text { were registered separately. Intraoperative incidences were recorded. The } \\
\text { follow-up was up to } 1 \text {-month, during which range of motion, superficial or } \\
\text { deep infection and wound dehiscence were assessed. } \\
\text { Results } \\
\text { There was a significant reduction in the capsule layer ( } 27 \text { sec, } p=0,02 \text { ) } \\
\text { and global time closure }(51 \text { sec, } p=0,01 \text { ) in the Q-group. No differences } \\
\text { were found in the subcutaneous layer ( } 24 \text { sec, } P=0,055 \text { ). There were more } \\
\text { intraoperative suture breakages in the Q-group, mainly in the } \\
\text { subcutaneous layer ( }<<0,001 \text { ). No differences in terms of dehiscence, } \\
\text { infection and ROM were observed at the } 1 \text {-month follow up. } \\
\text { Conclusions } \\
\text { BBS sutures allow for slightly faster wound closure than Vicryl during a } \\
\text { TKA. However the differences observed have minimal clinical } \\
\text { repercussions. Moreover, no differences in the infection rate (deep or } \\
\text { superficial), dehiscence or ROM were found. }\end{array}$ \\
\hline
\end{tabular}

\section{SCHOLARONE}

Manuscripts 
1 Title: Barbed Sutures in TKA: Are they really useful? A Randomized Controlled Trial

2

3

4

5

6

7

9 related to them.

\section{Methods}

11 It was a randomized controlled trial (RCT). Eighty-five patients undergoing primary TKA were assigned to receive traditional closure with Vycril (V-Group) or with BBS (Q-group). The

18 were assessed.

\section{Results}

There was a significant reduction in the capsule layer (27 sec, p=0,02) and global time closure observed at the 1-month follow up.

exclusion criteria were significant coronal deformity, flexion contracture or the need for stem and/or augmentation. The closure time for the capsule and subcutaneous layer were registered separately. Intraoperative incidences were recorded. The follow-up was up to 1month, during which range of motion, superficial or deep infection and wound dehiscence (51 sec, p=0,01) in the Q-group. No differences were found in the subcutaneous layer (24sec, $\mathrm{P}=0,055)$. There were more intraoperative suture breakages in the Q-group, mainly in the subcutaneous layer $(\mathrm{p}<0,001)$. No differences in terms of dehiscence, infection and ROM were

\section{Conclusions}


BBS sutures allow for slightly faster wound closure than Vicryl during a TKA. However the differences observed have minimal clinical repercussions. Moreover, no differences in the infection rate (deep or superficial), dehiscence or ROM were found.

\section{Keywords:}

Barbed suture, Total Knee Arthroplasty, Closure time TKA (1)

\section{INTRODUCTION}

Surgical time and effective wound closure are both important elements in preventing complications in Total Knee arthroplasty (TKA) 1. Barbed sutures have recently been investigated for their capacity to reduce closure time and thus the cost of surgery in TKA ${ }^{2-3-4-}$ 5-6. They make it possible for the surgeon to perform a knotless running suture with the same mechanical properties as conventional sutures ${ }^{7}$. Few reports describe bi-directional barbed sutures (BBS) as an effective tool to perform a rapid and effective wound closure with similar results to conventional sutures in terms of functional and complication outcomes ${ }^{8-9-10-11}$. The safety of this new kind of suture has recently been questioned due to reports of an elevated infection rate with respect to the conventional suture ${ }^{12-13}$.

Published literature provides little evidence to support using this new tool when performing TKA.

\subsection{Purpose}

The aim of the study was to test the effectiveness of BBS in reducing closure time. The secondary objectives were to assess the infection rate, wound dehiscence, functional outcomes and incidences occurring during surgery. The initial hypothesis was that the closure time was not influenced by the type of suture used. 


\subsection{Study population}

53 A randomized controlled blind trial (RCT) was carried out between January and July 2016 in

54 one single institution. Approval was given by institution's ethics committee (2015/6529).

55 Patients programmed to receive primary TKA were enrolled after signing informed consent.

56 Patients with a previous knee surgery or necessitating a tibial or femoral stem were excluded.

57 Other exclusion criteria were a coronal deformity over 15 degrees or a flexion contracture 58 superior to 20 degrees.

\subsection{Surgical technique}

61 A cemented TKA was implanted in a standard way with the patient lying supine and a thigh tourniquet was applied. A medial parapatellar approach was used in all cases. The patella was always substituted in this work. After closure, the wound was finally sealed with metallic staples in all cases. The knee was immobilized in extension with a removable splint. Articular aspirating drainage was left in place for 24 hours.

All surgeries were performed by 4 different knee surgeons. Three different TKA models were implanted during the study period: Triathlon ${ }^{\circledR}$ - Stryker (Mahwah, New Jersey, United States), Genutech ${ }^{\circledR}$ - Surgival (Valencia, España), U2®- United Orthopedic Corporation (Taiwan).

Patients from both groups followed the same rehabilitation protocol. It included continuous passive motion beginning the day after surgery that lasted until hospital discharge at around the fourth postoperative day. All of them had a minimum of 10 physiotherapy sessions in the outpatient clinic.

\subsection{Randomization}


Patients were randomly assigned to receive a standard wound closure (V-group) or with barbed sutures (Q-Group). The randomization process was carried out with the closed envelope system just before starting the closure process. Closure was then performed simultaneously by the chief surgeon and the first assistant starting from the centre of the wound and running to opposite ends. Previous to the study, all the participants went through a training period with BBS with at least 10 cases. In the V-group, polyglactin 910 (VycrilEthicon, Somerville, New Jersey, United States) was used with interrupted sutures while BBS (Quill- Angiotech, Vancouver, Canada) was used in a continuous running fashion in the Qgroup. Size 2 sutures were used for the capsular layer and size 2-0 for the subcutaneous layer in both groups. The capsule and subcutaneous layer closure times were registered separately and together (global time). The incidence of suture rupture was also collected.

Range of motion (ROM) at 1-month was measured. Additionally, any superficial or deep infection as well as dehiscence of the wound was documented. The diagnosis of superficial wound infection was established using the CDC criteria for superficial incisional SSI 14-15, revised in January $2016^{16}$. Follow-up was up to 1-month.

\subsection{Statistical analyisis}

The statistical analysis was performed using the SPSS v22 software package. The Student's tTest and Chi-Square test were used to analyse the data and $\mathrm{P}<0.05$ was considered to be significant. A power analysis was previously done anticipating a $10 \%$ loss to follow up. The sample size was calculated based on the results of previous studies ${ }^{3-9-10-17}$. A minimum of 33 patients in each group was considered sufficient to arrive at consistent findings. 
Eighty-five patients were enrolled and randomized into two groups. They were the V-group

101

102

103

104

105

106

107

108

109

110

111

112

113

114

115

116

117

118

119

120

121

122

123

with 44 patients and the Q-group with 41 . The demographic characteristics of the sample are summarized in Table 1. Both groups were comparable relative to the basal characteristics. There were no patients lost to follow-up.

The closure of the capsular layer was superior in the V-group than the Q-group $(\mathrm{p}=0.02)$. However, this difference was not observed in the subcutaneous layer (n.s.). The global surgical wound closure time (capsular plus sub-cutaneous) was also shorter in the Q-group than in the V-group ( $\mathrm{p}=0.01)$. All these results are summarized in the Table 2. This table also shows the different complications observed in both groups.

\section{DISCUSSION}

The main finding of this study is that BBS significantly reduce closure time following a TKA when is compared to Vicryl sutures. In that sense, our initial hypothesis was not confirmed. A second finding of this study was that the complications observed do not depend on the type of suture used.

Different publications have reported on the effectiveness of BBS in shortening closure time after a TKA ${ }^{3-4-5-9}$. Chan et al ${ }^{2}$, with a sample similar to our study group, found an average reduction time of about 4 minutes while Gilliland et al. reported a reduction of 4.6 minutes in the overall closure time ${ }^{2}$. Smith et al. even observed a difference up to 9 minutes in both groups ${ }^{17}$. On the other hand, a meta-analysis conducted by Meena et al did not find differences in total procedure time using BBS in comparison to conventional sutures ${ }^{9}$. The authors suggested that these results were due to the different levels of training of the surgeons and because additional factors may affect the entire duration of the procedure ${ }^{9-17}$. 
124 Wound closure in the BBS group was 51 seconds faster than in the Vicryl group. It is a smaller

125 reduction than described in other studies, which may be due to the shorter global closure

126 time ${ }^{3-17-18}$. The presence of 2 surgeons closing the wound simultaneously may justify this

127 finding. However, Gilliland et al. and Sah used a similar protocol but longer closure times ${ }^{3-18}$.

128 On the other hand, it is controversial as to whether the difference observed (51 sec) is

129 clinically significant. The authors do not feel that such a reduction in the time needed to

130 perform a TKA has any repercussion on the result of this procedure.

131 The results in terms of ROM at 1-month showed no differences between traditional sutures

132 and BBS. The same findings were reported by Chan et al 2 . Slightly better results would be

133 expected from BBS because running sutures are supposed to share out mechanical forces

134 better, but again many aspects are coming together to condition better or worse ROM.

135 It has been suggested that barbed monofilament sutures decrease bacterial adhesion in 136 comparison to traditional sutures. Theoretically, this fact could protect against infection ${ }^{19}$. In

137 the present study, wound related complications and the infection rate were similar in both 138 groups. Neither were any differences reported in 2 meta-analyses and other published RCT ${ }^{2-}$

139 3-8-9. However, Campbell et al. described a higher incidence of wound problems (19,5\%) that 140 included superficial and deep infections and dehiscence using the barbed suture for skin 141 closure ${ }^{12}$. Chawla et al. also reported an odds ratio of wound infection of about 22 with uni142 compartimental knee arthroplasty while describing 8 complications out of 333 patients in 143 which BBS were used ${ }^{13}$. Both studies are of a retrospective nature but are characterized by a 144 large sample size ${ }^{12-13}$.

145 Evident differences were found in intraoperative incidence using the barbed suture. Most of 146 them were suture breakage in the subcutaneous layer (16 in subcutaneous - 1 capsule $147 \mathrm{p}<0.000)$. The rate of suture rupture was higher in the Q-group than the V-group. In fact, no 148 problems were reported in this cohort with traditional sutures. Suture rupture is an aspect 
149

150

151

152

153

154

155

156

157

158

159

160

161

162

163

164

165

166

167

168

169

170

171

172

173

highlighted in a meta-analysis by Borzio et al. and in an RCT published by Gilliland et al in which $6 \%$ of the sutures ruptured intraoperatively ${ }^{3-8}$. The rate of suture breakage observed $(41 \%)$ is superior in our study. This fact could be due to less familiarity with this suture although each surgeon had used it for at least 10 cases before initiating this trial. The authors saw that BBS had a good strong sealing capacity in harder connective tissue like the capsule layer where barbs can also provide more effective anchorage. This is not as true as in the subcutaneous layer in which connective tissue is softer and efficient closure, in case of suture breakage, cannot be assured.

\subsection{Limitations}

This study is not free of limitations. One is that the number of sutures used for each patient was not recorded and neither was the price for the sutures used in each case. Regardless, cost analysis was not our goal. Although not only two different types of sutures but also two different techniques were used, the real goal of this research was to confirm if BBS could offer some advantages over traditional closure technique used in our institution. Another limitation is that the patients were followed for a limited time but it was enough time to observe the results related to the sutures or any complications. Moreover, the presence of more than four surgeons might have introduced a bias although it may have improved external validity.

\section{CONCLUSION}

Barbed sutures allow for a slightly shorter wound closure time in comparison to conventional interrupted sutures. No differences were found in the deep or superficial infection rate, wound dehiscence and ROM between BBS and Vycril sutures. Finally, an elevated number of intra-operative suture breakages is to be expected when used in the subcutaneous layer. 
174 The results obtained here lead the authors not to recommend the use of barbed sutures in the 175 subcutaneous layer although it may be useful in the capsular layer.

176

177

178

\section{REFERENCES:}

179

180

181

182

183

184

185

186

187

188

189

190

191

192

193

194

195

196

197
1. Peersman G, Laskin R, Davis J, Peterson G E, Richart T. Prolonged operative time correlates with increased infection rate after total knee arthroplasty. HSS J 2006;2:702

2. Chan V W, Chan P, Chiu K, Yan C, Ng F. Does barbed suture lower cost and improve outcome in total knee arthroplasty? A randomized controlled trial. J Arthroplasty 2017 2017; 32(5):1474-7

3. Gilliland J, Anderson L, Barney J, Ross H L, Pelt C E, Peters C L. Barbed versus standard sutures for closure in total knee arthroplasty: a multicentre prospective randomized trial. J Arthroplasty 2014; 29 (9 Suppl):135-8

4. Levine B R, Ting N, Della Valle C J. Use of a barbed suture in the closure of hip and knee arthroplasty wounds. Orthopedics $2011 ;(9): \mathrm{e} 473-5$

5. Ting N T, Moric M M, Della Valle C J, Levine B R. Use of Knotless Suture for Closure of Total Hip and Knee Arthroplasties. J Arthroplasty 2012; 27: 1783-8

6. Maheshwari A V, Naziri Q, Wong A, Burko I, Mont M A, Rasquinha V J. Barbed sutures in total knee arthroplasty: are these safe, efficacious, and cost-effective? J Knee Surg $2015 ; 28: 151-6$

7. Vakil J J, O’Reilly M P, Sutter E G, Mears S C, Belkoff S M, Khanuja H S. Knee Arthrotomy Repair With a Continuous Barbed Suture - a Biomechanical Study. J Arthroplasty 2011; 26(5): 710-3 
8. Borzio RW, Pivec R, Kapadia B H, Jauregui J J, Maheshwari A V. Barbed Suture in total hip and knee arthroplasty: what is the evidence? A meta-analysis. Int Orthop ;40(2):225-231

9. Meena S, Gangary S, Sharma P, Chowdhury B. Barbed versus standard sutures in total knee arthroplasty: a meta-analysis. Eur J Orthop Surg traumatol 2015;25(6):1105-10

10. Zhang W, Xue D, Yin H, Xie H, Ma H, Chen E, Hu D, Pan Z. Barbed versus traditional 204 analysis. Sci Rep 2016; 6:19764

11. Stephens S, Politi J, Taylor B C. Evaluation of primary total knee arthroplasty incision closure with the use of continuous bidirectional barbed suture. Surg Techonol Int 2011;21:199-3

12. Campbell A L, Patrick Jr D A, Liabaud B, Geller J A. Superficial wound Closure Complications with Barbed Sutures Following Knee Arthroplasty. J Arthroplasty 2014;29: 966-9

13. Chawla H, Van der List J P, Frein N B, Henry M W, Pearle A D. Barbed Suture is Associated with Increased Risk of Wound Infection After Unicompartmental Knee Arthroplasty. J Arthroplasty 2016; 31(7):1561-7

14. Horan TC, Gaynes RP, Martone WJ, Jarvis WR, Emori TG. CDC definitions of nosocomial surgical site infections, 1992: a modification of CDC definitions of surgical wound infections. Infect Control Hosp Epidemiol 1992;13:606-8

15. Mangram AJ, Horan TC, Pearson ML, Silver LC, Jarvis WR. Guideline for prevention of surgical site infection, 1999. Centers for disease control and prevention (CDC) hospital infection control practices advisory committee. Am J Infect Control 1999; 27:97-132 16. NHSN (2016) Surgical site infecion (SSI) event. http://www.cdc. gov/nhsn/pdfs/pscmanual/9pscssicurrent.pdf 
17. Smith E L ,Disegna S T, Shukla P Y, Matzkin E G. Barbed Versus Traditional Sutures: Closure Time, Cost, and Wound Related Outcomes in Total Joint Arthroplasty. J Arthroplasty 2014;29: 283-7

18. Sah P. Is there an advantage to knotless barbed suture in TKA wound closure? A Randomized trial in simultaneous bilateral TKAs. Clin Orthop Relat Res 2015;473(6):2019-2017

19. Morris M R, Bergum C, Jackson N, Markel D C. Decreased bacterial adherence, biofilm formation, and tissue reactivity of barbed monofilament suture in an in vivo contaminated wound model. J Arthroplasty 2017;2(4):1272-9 DOI: 10.1016/j.arth.2016.11.010.

20. Wright R C, Gillis C T, Yacoubian S V, Raven R B, Falkinstein Y, Yacoubian S V. Extensor Mechanism Repair Failure With Use of Bidirectional Barbed Suture in Total Knee Arthroplasty. J Arthroplasty 2012;27 (7):1413e.1-4 
Table 1

\begin{tabular}{|llll|}
\hline & V-Group & Q-group & p \\
\hline Age (years) & $73.8 \pm 7.5$ & $74.2 \pm 8.2$ & n.s. \\
Gender (\%) & $31.7 \% \mathrm{M} / 68.3 \%$ & $38.6 \% \mathrm{M} \mathrm{/} \mathrm{61.4 \%}$ & \\
& $\mathrm{W}$ & $\mathrm{W}$ & n.s. \\
Weight $(\mathrm{kg})$ & $80.2 \pm 13.4$ & $75.9 \pm 15$ & n.s. \\
BMI $(\mathrm{kg} / \mathrm{m} 2)$ & $30.6 \pm 4.6$ & $30.2 \pm 5$ & n.s. \\
\hline
\end{tabular}

Table.1: Demographic data of both groups 
Table 2

\begin{tabular}{|c|c|c|c|}
\hline & V-Group & Q-group & $\mathrm{p}$ \\
\hline Capsule (min) & $3.39 \pm 0.9$ & $2.93 \pm 0.82$ & 0.019 \\
\hline Subcutaneous (min) & $3.76 \pm 0.86$ & $3.35 \pm 0.98$ & n.s. \\
\hline Global (min) & $7.15 \pm 1.38$ & $6.28 \pm 1.52$ & 0.01 \\
\hline Flexion $(\underline{o})^{*}$ & $102.4 \pm 12.41$ & $100.9 \pm 5.93$ & n.s. \\
\hline Extension $(\underline{o})^{*}$ & $-1.6 \pm 3.85$ & $1.5 \pm 3.79$ & n.s. \\
\hline Superficial infection & $1(2.3 \%)$ & $3(7.5 \%)$ & n.s. \\
\hline Deep infection & $0(0 \%)$ & $0(0 \%)$ & n.s. \\
\hline Dehiscence & $1(2.3 \%)$ & $2(5 \%)$ & n.s. \\
\hline Suture breakages & $0(0 \%)$ & $17(41 \%)$ & $<0.001$ \\
\hline
\end{tabular}

*= Range of motion measured at 1-month postoperatively

Table.2: Variables studied in both groups $( \pm \mathrm{SD})$ 